University of Nebraska - Lincoln

DigitalCommons@University of Nebraska - Lincoln

Faculty Publications, College of Dentistry

Dentistry, College of

September 2004

\title{
The Effects of a Commercial Aluminum Airpolishing Powder on Dental Restorative Materials
}

William W. Johnson

University of Nebraska Medical Center College of Dentistry, wwjohnson@unmc.edu

Caren M. Barnes

University of Nebraska College of Dentistry, cbarnes@unmc.edu

David A. Covey

University of Nebraska Medical Center College of Dentistry, dcovey@unmc.edu

Mary P. Walker

University of Missouri-Kansas City School of Dentistry, walkermp@umkc.edu

Judith A. Ross

University of Tennessee Health Science Center College of Dentistry, jross@utmem.edu

Follow this and additional works at: https://digitalcommons.unl.edu/dentistryfacpub

Part of the Other Medicine and Health Sciences Commons

Johnson, William W.; Barnes, Caren M.; Covey, David A.; Walker, Mary P.; and Ross, Judith A., "The Effects of a Commercial Aluminum Airpolishing Powder on Dental Restorative Materials" (2004). Faculty Publications, College of Dentistry. 2.

https://digitalcommons.unl.edu/dentistryfacpub/2

This Article is brought to you for free and open access by the Dentistry, College of at DigitalCommons@University of Nebraska - Lincoln. It has been accepted for inclusion in Faculty Publications, College of Dentistry by an authorized administrator of DigitalCommons@University of Nebraska - Lincoln. 
Published in Journal of Prosthodontics 13:3 (September 2004), pp. 166-172; doi 10.1111/j.1532-849X.2004.04026.x Copyright (C) 2008

American College of Prosthodontists; published by Blackwell/John Wiley \& Sons. Used by permission.

http://www3.interscience.wiley.com/journal/118544241/home

Supported in part by grants from Dentsply Preventive and the UNMC College of Dentistry.

Presented at the AADR/IADR annual meeting, San Antonio, Texas, March 13, 2003.

Accepted February 20, 2004; published online September 2, 2004.

\title{
The Effects of a Commercial Aluminum Airpolishing Powder on Dental Restorative Materials
}

\author{
William W. Johnson, DDS, MS; ${ }^{*}$ Caren M. Barnes, RDH, MS; ${ }^{\dagger}$ David A. Covey, DDS, MS; ${ }^{\ddagger}$ \\ Mary P. Walker, DDS, MS, PhD; $\$$ and Judith A. Ross, DMD, MS \\ * Associate Professor, Department of Adult Restorative Dentistry, \\ University of Nebraska Medical Center College of Dentistry \\ † Professor, Department of Surgical Specialties, Coordinator of Clinical Research, \\ University of Nebraska Medical Center College of Dentistry \\ $\ddagger$ Associate Professor, Department of Adult Restorative Dentistry, \\ University of Nebraska Medical Center College of Dentistry \\ $\S$ Associate Professor and Director of Dental Biomaterials, Department of Restorative Dentistry, \\ UMKC School of Dentistry (Formerly: Assistant Professor, Department of Adult Restorative Dentistry, \\ University of Nebraska Medical Center College of Dentistry) \\ I Associate Professor, Restorative Department, \\ University of Tennessee Health Science Center College of Dentistry \\ Corresponding author: C. M. Barnes, RDH, MS, \\ University of Nebraska Medical Center College of Dentistry, Department of Surgical Specialties, \\ 40th \& Holdrege, Lincoln, Nebraska 68583-0740; email cbarnes@unmc.edu
}

\begin{abstract}
Introduction: JET-Fresh ${ }^{\mathrm{TM}}$ (Dentsply International, York, PA), a new airpolishing powder that contains aluminum trihydroxide as the abrasive agent, has been introduced for use with Prophy-Jet ${ }^{\mathrm{TM}}$ (Dentsply International).

Purpose: The aim of this study was to investigate the surface effects of aluminum trihydroxide airpolishing powder on a series of restorative materials.

Materials and Methods: A total of 6 Class V preparations with enamel margins were created on the buccal surface of extracted human molars. The preparations were restored with Type III gold, a high copper spherical alloy amalgam, porcelain, a lightactivated hybrid composite material, a light-activated microfilled composite material, and a light-activated, resin-modified glass ionomer material. The distal half of each restoration was covered with fiberglass tape and the mesial half subjected to treatment with the aluminum trihydroxide powder via the Prophy-Jet ${ }^{\mathrm{TM}}$ for 5 seconds. After removal of the protective tape, the buccal surface of each treated tooth was replicated with impression material. Replicas were generated using epoxy resin and prepared for evaluation with scanning electron microscopy.

Results: The aluminum trihydroxide produced surface alterations that were apparent visually and when viewed by a scanning microscope of the hybrid and microfilled composites and the glass ionomer restorations. The surfaces of the amalgam and gold restorations were altered, but not to the extent that the resin-based materials were. No disruption of the surface characterization of the porcelain was detected; however, with gold and porcelain materials, the aluminum trihydroxide removed notable amounts of the luting cements (the results are consistent with the data gathered with sodium bicarbonate powder).

Conclusion: Aluminum trihydroxide as the abrasive agent in an airpolishing system should be avoided on resin composites, resin-modified composites, and around the margins of cemented restorations.
\end{abstract}

Keywords: airpolishing, polishing dental restorations, aluminum trihydroxide polishing powder

PROPHY-JET ${ }^{\mathrm{TM}}$ (Dentsply International, York, PA) is an airpolishing system that utilizes air, water, and sodium bicarbonate to deliver a controlled stream that propels specially processed sodium bicarbonate particles to the tooth surface for stain and plaque removal. Research studies comparing this to 
the traditional rubber cup and pumice polishing procedure have established that the Prophy-Jet ${ }^{\mathrm{TM}}$ system is less time consuming, less abrasive, and requires less physical exertion by the operator while it generates little or no heat. ${ }^{1,2}$

When the Prophy-Jet ${ }^{\mathrm{TM}}$ system of airpolishing was first introduced, there were well-founded concerns regarding the effect the airpolishing would have on dental restorative materials. Cooley and Lubow evaluated the effect of an airpolishing system that utilized sodium bicarbonate powder on the surface characteristics of various restorative materials. They found that the composite resins underwent the greatest change in roughness. ${ }^{3,4}$ No surface alteration was seen with porcelain, while the change in surface roughness of the metals studied was not clinically significant. Barnes et al. ${ }^{1}$ investigated the effects of Prophy-Jet ${ }^{\mathrm{TM}}$ utilizing sodium bicarbonate particles on microfilled composite material, a large particle composite material, amalgam, and gold. The time of exposure of each of these materials was 5 seconds, simulating 10 years of recall polishing procedures. ${ }^{1}$ The findings from the study indicated that the sodium bicarbonate particles rapidly removed the surface of the composite resins, exposing the composite filler particles. Notably, there was no disruption of surface integrity or margins of the amalgam restorations or gold restorations. However, the sodium bicarbonate removed significant amounts of cement around the margins of the gold restorations. The findings of Barnes, Cooley, and Lubow mirror those found by other investigators. ${ }^{5-12}$

Traditionally, the Prophy-Jet ${ }^{\mathrm{TM}}$ airpolishing system has utilized a specially processed sodium bicarbonate powder delivered in a controlled stream of air and water to the tooth surface for stain and plaque removal. The sodium bicarbonate particles are less abrasive than particles found in pumice or commercially prepared polishing pastes. ${ }^{13}$

The Mohs hardness number for specially formulated sodium bicarbonate utilized for stain and plaque removal is 2.5. ${ }^{13}$ Even though the Mohs hardness number is low, the powder utilized in the airpolishing unit (Prophy-Jet ${ }^{\mathrm{TM}}$ ) has proven detrimental to the surface characterization and integrity of composites, glass ionomers, and gold.

An alternative to the sodium bicarbonate air- pol- ishing agent has recently been introduced. The alternative polishing powder, JET-Fresh ${ }^{\mathrm{TM}}$, contains the active and widely used polishing agent aluminum trihydroxide. The Mohs hardness number for aluminum trihydroxide is 4.0. ${ }^{13}$ Given that aluminum trihydroxide is a harder polishing agent than sodium bicarbonate, it is hypothesized that JET-Fresh ${ }^{\mathrm{TM}}$ will also affect the surface characterization and integrity of many restorative materials. The purpose of this study is to assess the effect of JET-Fresh ${ }^{\mathrm{TM}}$ on common dental restorative materials.

\section{Materials and Methods}

The study was performed on 6 extracted human teeth that had been stored in distilled water since the time of extraction. The teeth selected for the investigation were free of caries and did not have craze lines or other enamel defects. One Class V preparation with dimensions of $5 \mathrm{~mm}$ (mesial-distal) $\times 2 \mathrm{~mm}$ (occlusal- gingival) $\times 2 \mathrm{~mm}$ deep was prepared on each of the teeth.

Six different materials were used to restore the Class V preparations. The materials utilized are listed in Table 1. Each restoration was shaped to mimic the original contours of the unprepared tooth. The 2 composite resin materials and the resin-modified glass ionomer material were placed following the manufacturers' instructions, utilizing the companion bonding and/or conditioning systems supplied with the restorative kits. The amalgam was triturated according to the manufacturer's instructions using a Varimix II amalgamator (Dentsply Caulk, Milford, DE). No varnish was placed on the preparation prior to condensation of the dental amalgam. The amalgam restoration was allowed to set for 24 hours prior to final polishing. The cast gold inlay was cemented into the preparation using FujiCEM ${ }^{\mathrm{TM}}$ (GC America, Inc., Alsip, IL). The porcelain inlay was cemented with Nexus resin cement (Kerr Corp., Orange, CA). All of the restorations, with the exception of the porcelain inlay, were polished with PoGo ${ }^{\circledR}$ diamond micropolishers (Dentsply Caulk). The restorations were polished until the surfaces appeared to be uniformly smooth and free of scratches or voids. The porcelain inlay was glazed in the laboratory and no further polishing was done. The completed restorations were stored for 1 week in distilled water at $37^{\circ} \mathrm{C}$ prior to subjecting them to airpolishing.

The distal half of each restoration was covered with fiberglass tape. The exposed surface of the restoration and surrounding tooth structure was submitted to treatment with the Prophy-Jet ${ }^{\mathrm{TM}}$ (Dentsply International) airpolishing system, utilizing JET-Fresh ${ }^{\mathrm{TM}}$ polishing powder. In each case, the orifice of the nozzle was held approximately $4 \mathrm{~mm}$ from the restored surface and a constant circular motion was used. The air pressure at the nozzle tip was held 
Table 1. Materials Used to Restore the Class V Preparations

\begin{tabular}{lll}
\hline Material & Description & Manufacturer \\
\hline Gold Inlay & Oro B-2 Gold & Dentsply Ceramco, Burlington, NJ \\
Dispersalloy ${ }^{\circledR}$ & High copper, admixed amalgam & Dentsply Caulk, Milford, DE \\
Finesse ${ }^{\circledR}$ & Low fusing porcelain & Dentsply Ceramco, Burlington, NJ \\
Esthet $\bullet X^{\mathrm{TM}}$ & Micro-matrix composite resin & Dentsply Caulk, Milford, DE \\
Filtek ${ }^{\mathrm{TM}}$ Z-250 & Hybrid composite resin & 3M ESPE, Minneapolis, MN \\
Fuji II LC v & Resin-modified glass Ionomer & GC America, Chicago, IL \\
\hline
\end{tabular}

at a constant $40 \mathrm{psi}$. The time of exposure for all samples was 5 seconds, which represents approximately 10 years of 6-month recall exposures. ${ }^{13-15}$

After the fiberglass tape was removed, the entire surface was washed with a spray of water and carefully air-dried. The entire buccal surface of each tooth was replicated with a hydrophilic polyvinylsiloxane impression material (Examinix, GC America). The second- stage replicas were generated using an epoxy resin (Bueler ${ }^{\circledR}$ Epoxide, Lake Bluff, IL) and sputter coated with gold/palladium in preparation for evaluation with scanning electron microscopy (SEM). Each specimen was examined and photographed with SEM at $40 \times, 150 \times$, and $500 \times$. The photomicrographs were examined by 2 independent evaluators for differences between the control surface and the side treated with the air- polishing system. In addition, each clinical surface was evaluated under clinical conditions, which included an operating light and $2.5 \times$ magnification for changes in surface reflectivity. In each instance, the control (untreated) side of the restoration was compared to the surface treated with the airpolishing unit. The evaluators decided whether the abrasion on the treated surface was slight, moderate, or severe compared to the control side.

\section{Results}

The effect of the aluminum trihydroxide utilized in the airpolishing system on the restorative materials is illustrated in a series of scanning electron micrographs. The effect of the polishing particles after 5 seconds of exposure on the surface of Esthet $\bullet X^{\mathrm{TM}}$ is shown in Figures 1a-c. For the purpose of orientation, the left side of the photomicrographs is the untreated surface. For all levels of magnification there is apparent alteration of the surface of the material, which appears to be uniform across the treated area.

The effect of the aluminum trihydroxide airpolishing on microfilled composite material (Filtek A110) mirrors that of the hybrid composite materials and can be seen in Figures 2a-c; however, the amount of microfilled composite resin removed appeared greater with residual microcracks.

Glass ionomer material treated with the aluminum trihydroxide airpolishing can be seen in Figures 3ac. The damage to the surface characterization of the glass ionomer material appears to be greater than the hybrid composite material, but not as severe as that to the microfilled material.

Dispersalloy amalgam was also treated and the results can be seen in Figures 4a-c. Notably, there appears to be surface removal of material that is especially apparent in Figure 4a. This surface alteration was not as severe as that seen in the glass ionomer or resin-based restorative materials. Clinically, the aluminum trihydroxide airpolishing produced a matte finish on the polished amalgam surface.

Indirect restorative materials treated with the aluminum trihydroxide polishing system can be seen in Figures 5 and 6. Type III gold is represented in Figures $5 \mathrm{a}-\mathrm{c}$. As with amalgam, there is a change in the surface characterization, but not to the extent seen in the resin-based materials. Clinically, the airpolishing produced a matte finish on the gold. Importantly, there is a notable removal of resin cement at the interface of the restoration. The porcelain treated in this study, Finesse, can be seen in Figures $6 a$ and b. There appears to be no disruption in the surface characterization, but the removal of the luting agent at the margin is acute.

\section{Discussion}

While airpolishing is quite effective in removing stains and dental plaque from tooth surfaces, ${ }^{16-21}$ it must be used with care. Regardless of the polishing agent used, whether sodium bicarbonate or aluminum trihydroxide, the use of these agents should be avoided on dental restorative materials. The effects of aluminum trihydroxide as the abrasive agent in airpolishing on resin composite materials and glass 


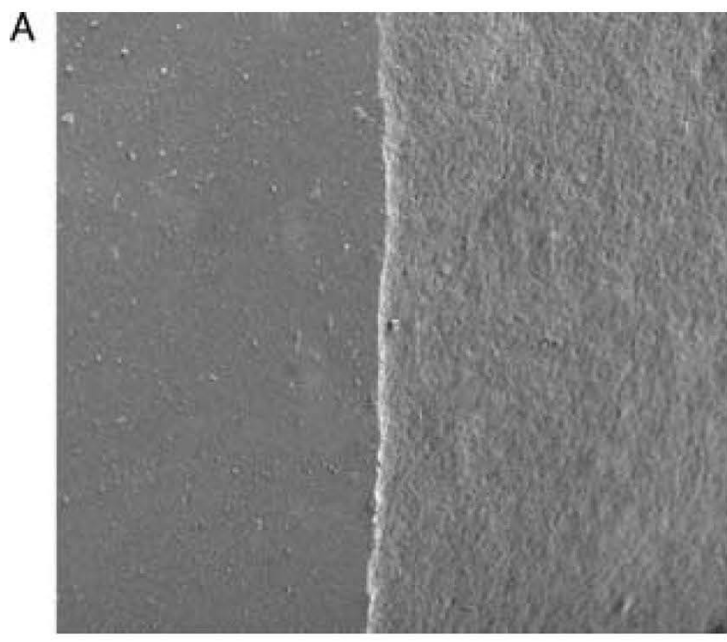

B

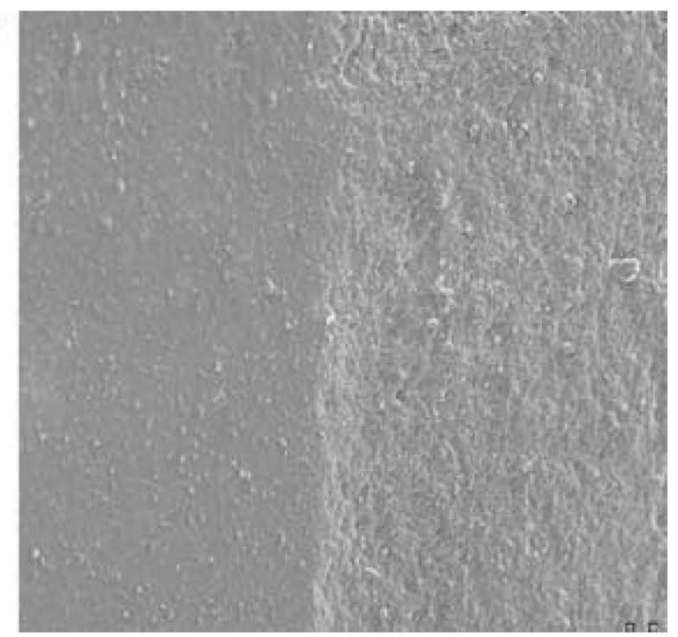

$\mathrm{C}$

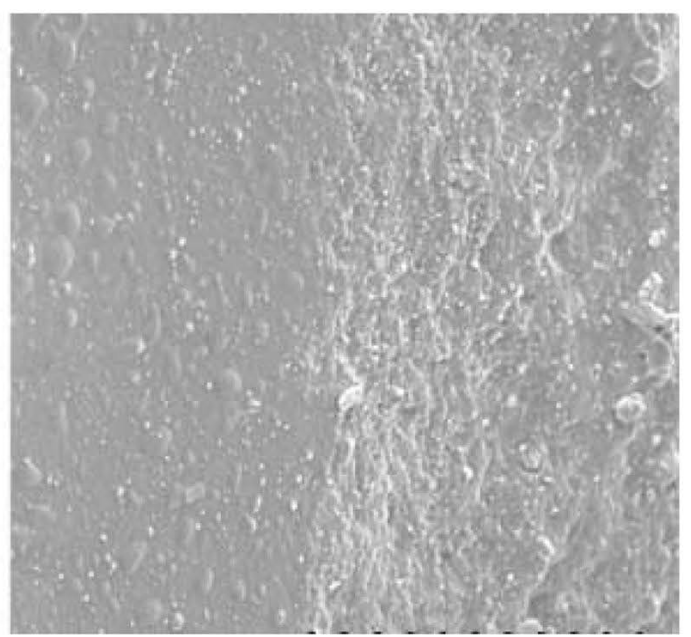

Figure 1. (A) SEM photograph of a replica of EsthetX at 40x. The left half of the restoration is the control surface, the right half is the treated surface. (B) SEM photograph of a replica of EsthetX at 150x. The left half of the restoration is the control surface, the right half is the treated surface. (C) SEM photograph of a replica of EsthetX at 500x. The left half of the restoration is the control surface, the right half is the treated surface.

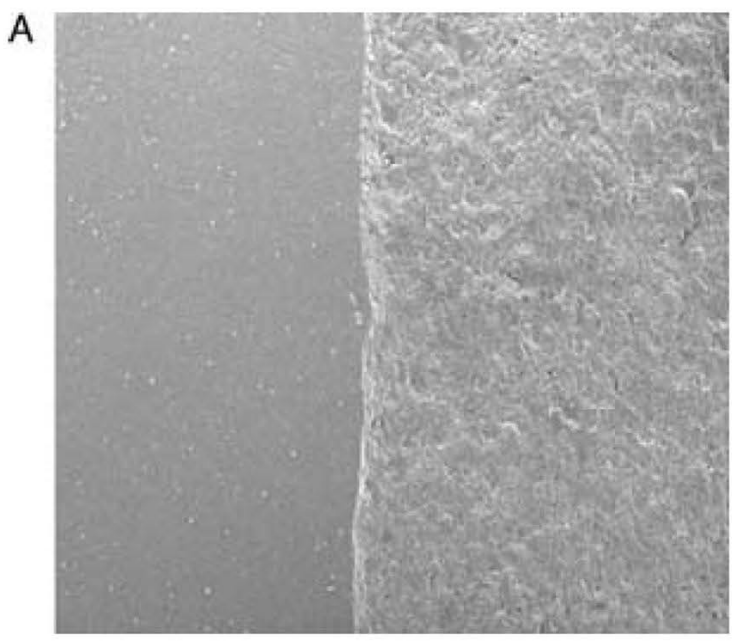

$\mathrm{B}$

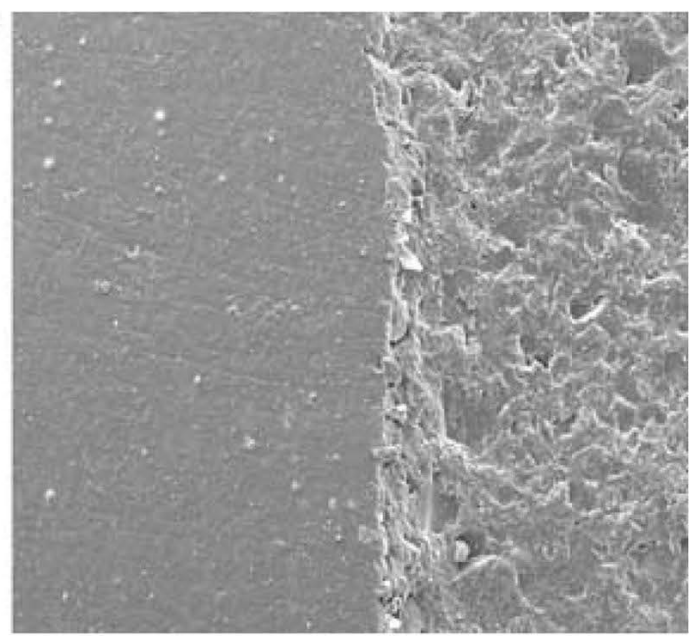

C

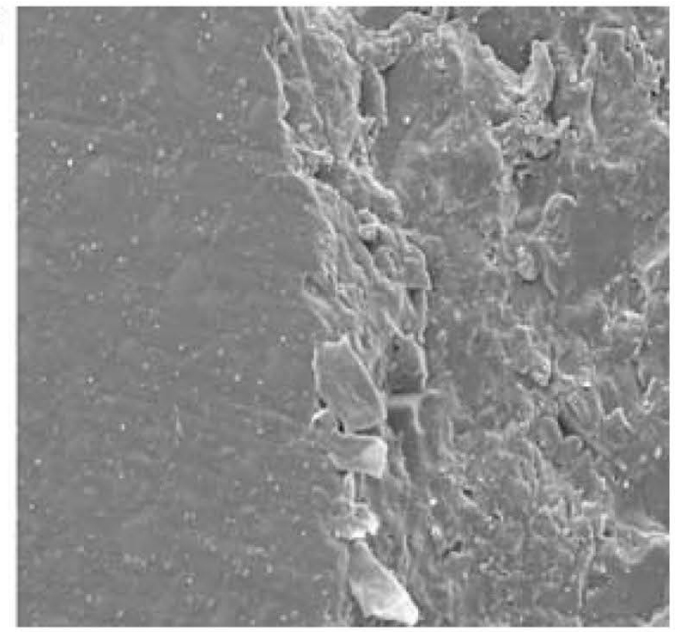

Figure 2. (A) SEM photograph of a replica of Filtek A 110 at $40 \mathrm{x}$. The left half of the restoration is the control surface, the right half is the treated surface. (B) SEM photograph of a replica of Filtek A 110 at 150x. The left half of the restoration is the control surface, the right half is the treated surface. (C) SEM photograph of a replica of Filtek A 110 at 500x. The left half of the restoration is the control surface, the right half is the treated surface. 
A

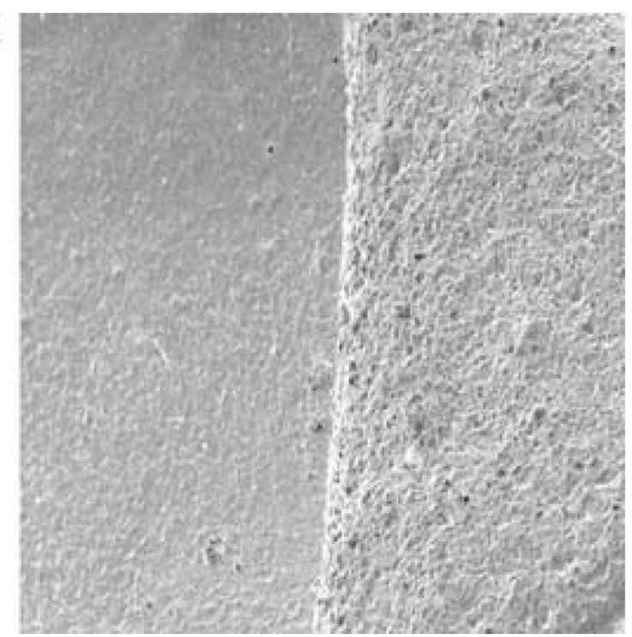

B

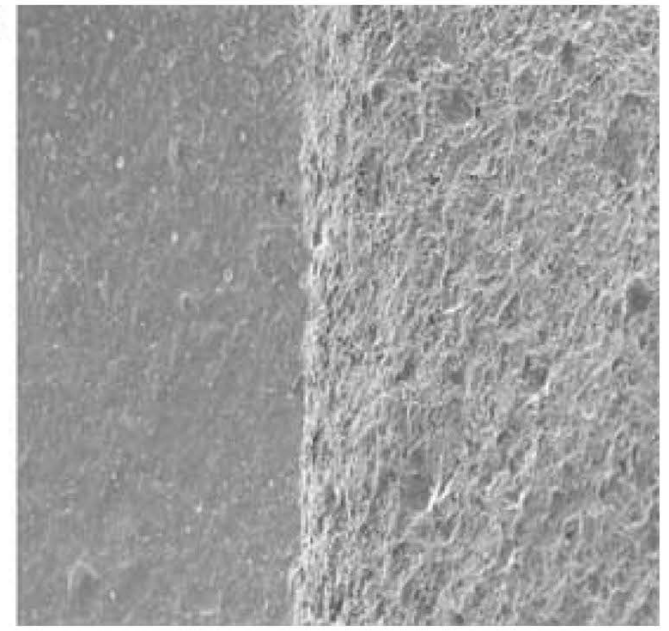

C

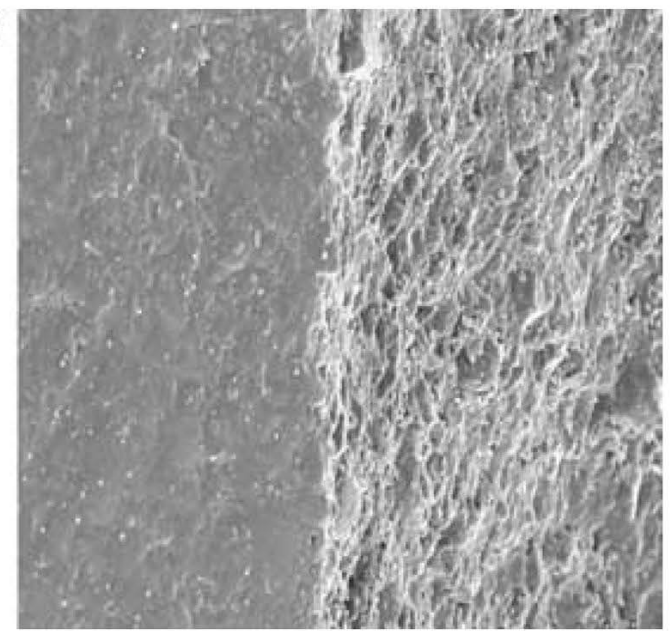

Figure 3. (A) SEM photograph of a replica of Fuji II LC at 40x. The left half of the restoration is the control surface, the right half is the treated surface. (B) SEM photograph of a replica of Fuji II LC at 150x. The left half of the restoration is the control surface, the right half is the treated surface. (C) SEM photograph of a replica of Fuji II LC at 500x. The left half of the restoration is the control surface, the right half is the treated surface.
A

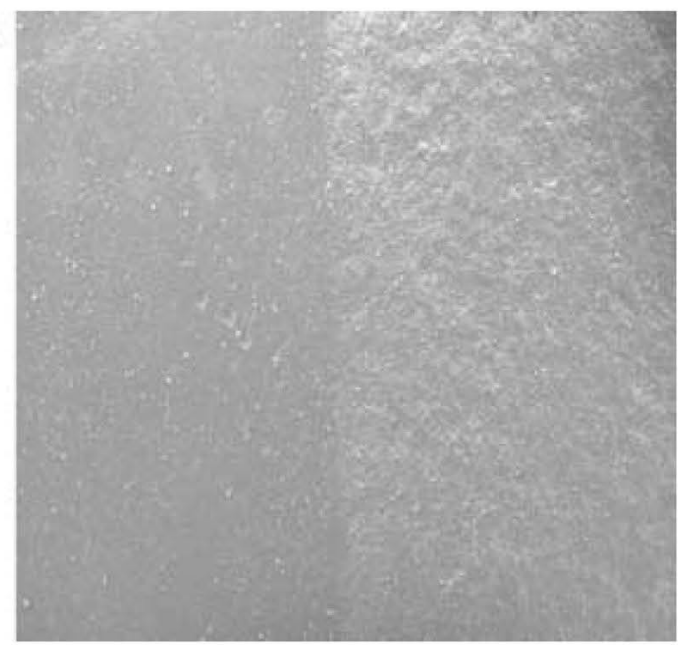

B

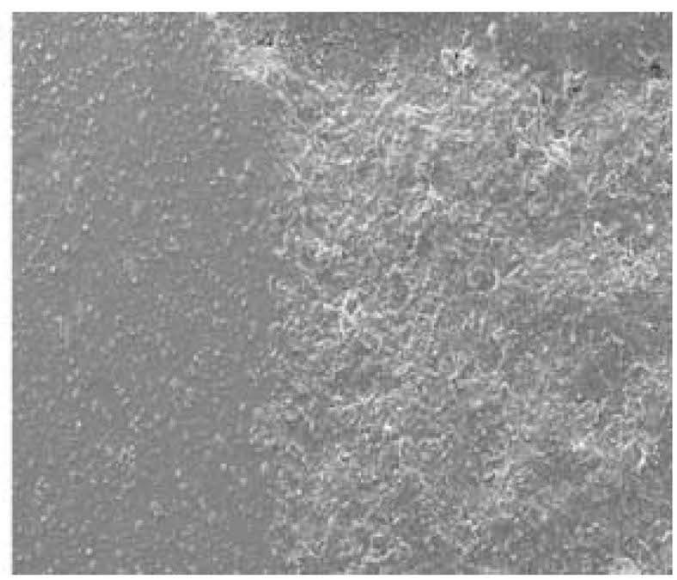

C

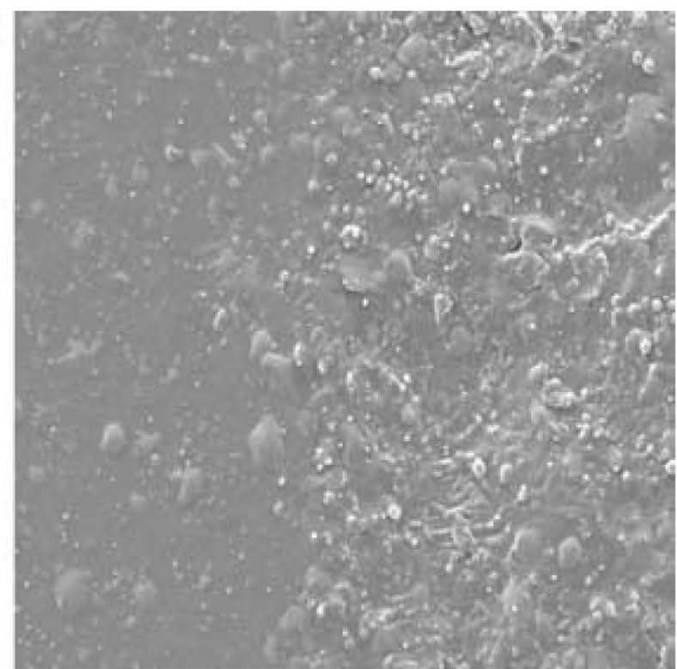

Figure 4. (A) SEM photograph of a replica of Dispersalloy at 40x. The left half of the restoration is the control surface, the right half is the treated surface. (B) SEM photograph of a replica of Dispersalloy at 150x. The left half of the restoration is the control surface, the right half is the treated surface. (C) SEM photograph of a replica of Dispersalloy at 500x. The left half of the restoration is the control surface, the right half is the treated surface. 


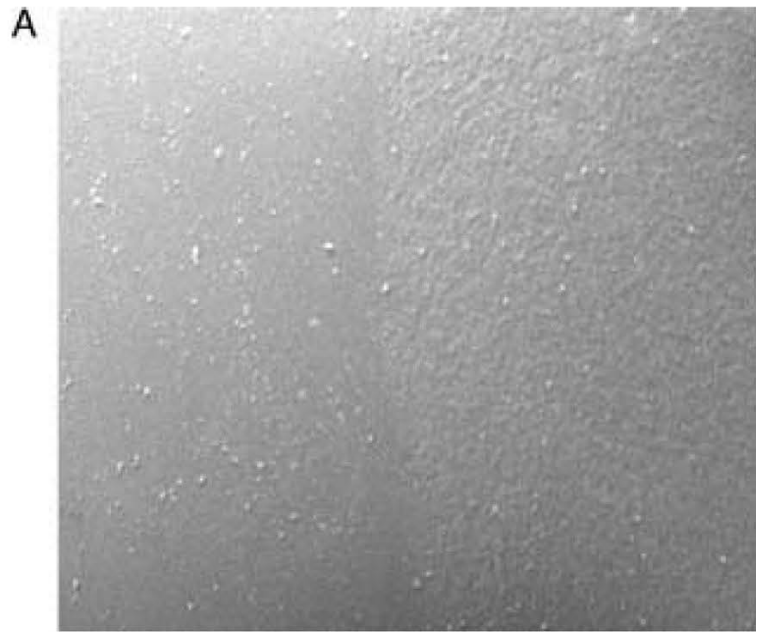

B
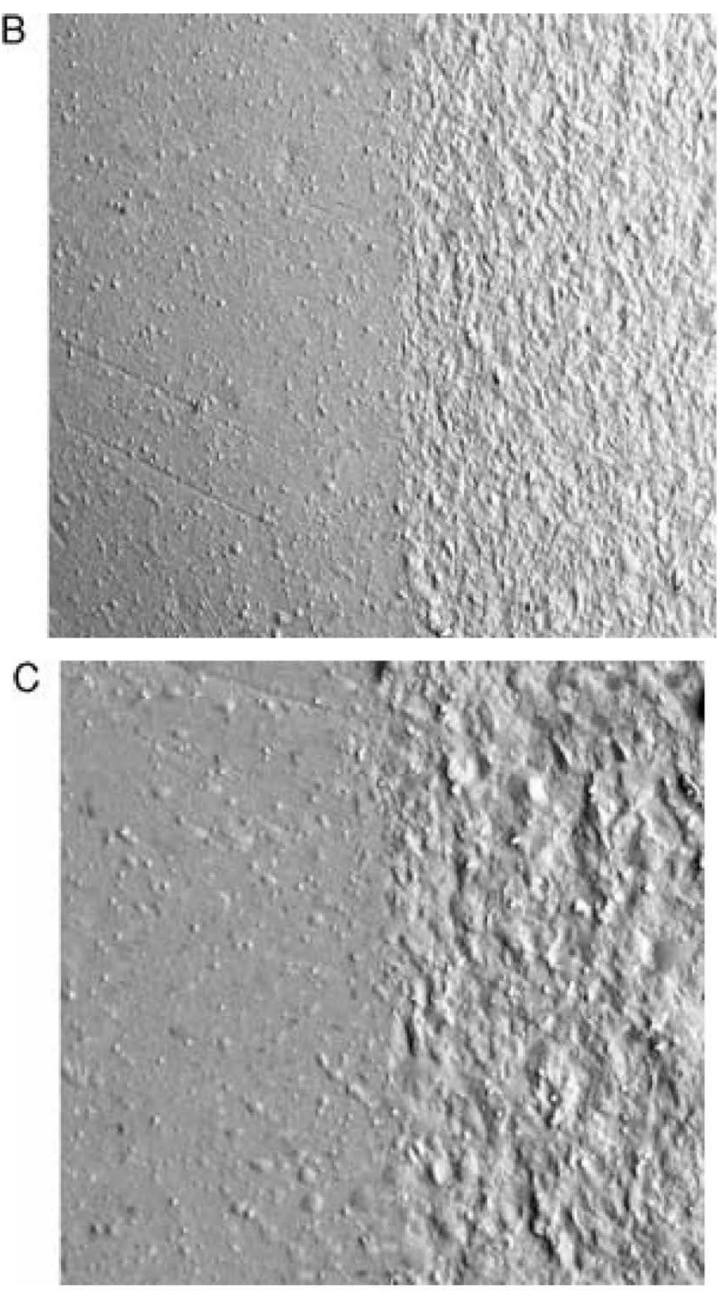

Figure 5. (A) SEM photograph of a replica of Type III gold at $40 x$. The left half of the restoration is the control surface, the right half is the treated surface. Note the removal of the luting agent on the treated side of the restoration. (B) SEM photograph of a replica of Type III gold at 150x. The left half of the restoration is the control surface, the right half is the treated surface. (C) SEM photograph of a replica of Type III gold at $500 x$. The left half of the restoration is the control surface, the right half is the treated surface.

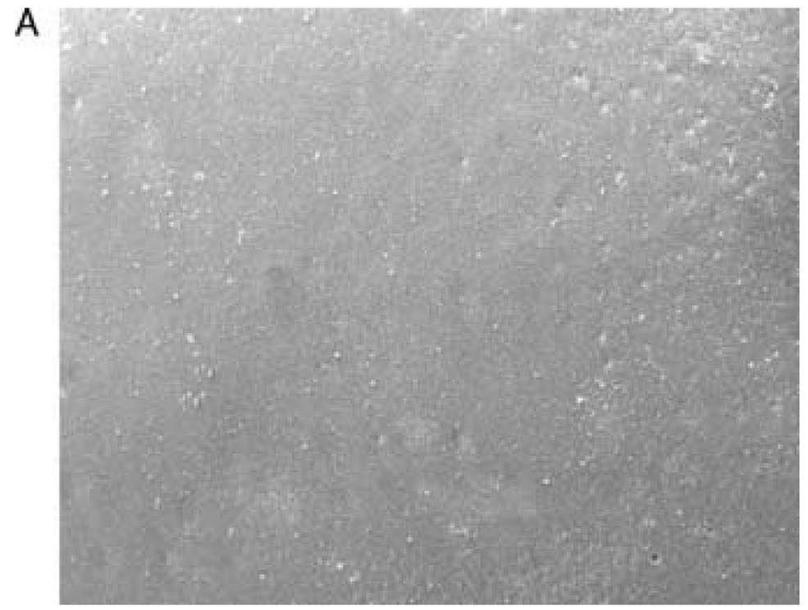

B

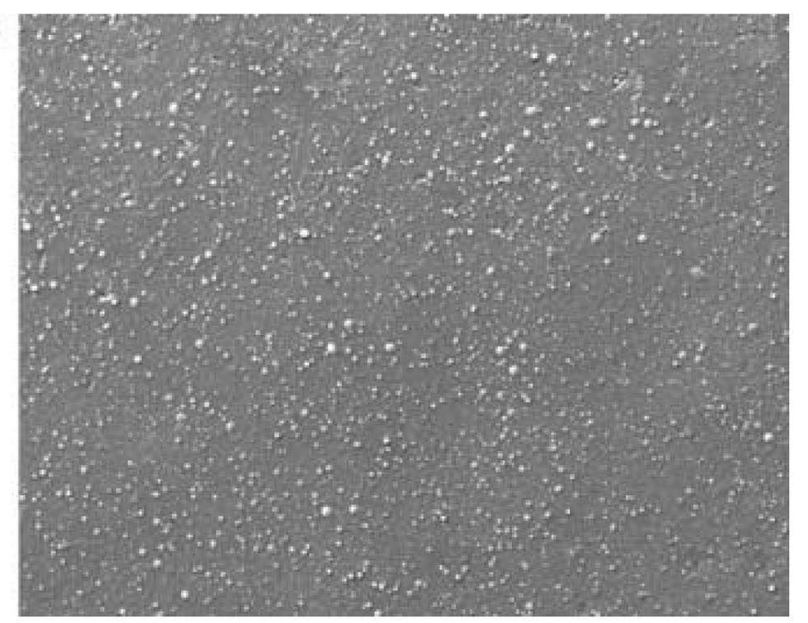

Figure 6. (A) SEM photograph of a replica of Finesse porcelain at $40 \mathrm{x}$. The left half of the restoration is the control surface, the right half is the treated surface. (B) SEM photograph of a replica of Finesse porcelain at 150x. The left half of the restoration is the control surface, the right half is the treated surface.

ionomers are no different than the effects of sodium bicarbonate. ${ }^{1-10}$ Both agents remove the resin and expose the filling particles, thus damaging the surface characterization of the restoration. The use of aluminum trihydroxide and sodium bicarbonate in airpolishing is also contraindicated for use on cast restorations. ${ }^{1,4}$ These agents produce a matte finish on gold clinically, but do not alter the surface characterization of porcelain. However, airpolishing agents quickly remove the luting cements and render the cast restorations compromised and vulnerable to plaque retention.

The effects of airpolishing with aluminum trihydroxide or sodium bicarbonate on restorative materials mimic those seen with traditional rubber cup polishing with a commercial prophylaxis paste. Com- 
mercially available prophylaxis pastes indicated for tooth polishing are not indicated for use on dental restorative materials. ${ }^{22}$ They produce deep irregular scratches in resin composites and glass ionomers, damaging the surface. Similarly, commercial prophylaxis pastes will produce a matte finish on amalgam and gold restorations; however, they do not alter the surface of the luting cements in a manner that is clinically significant. ${ }^{22,23}$

\section{Conclusions}

This in vitro study investigated the effects of aluminum trihydroxide utilized in an airpolishing system (Prophy-Jet ${ }^{\mathrm{TM}}$ ) on hybrid and microfilled composites, glass ionomers, porcelain, gold, and amalgam. Within the limitations of this study the following conclusions were drawn:

1. Use of aluminum trihydroxide as the abrasive agent in an airpolishing system should be avoided on luting cements, resin composites, and glass ionomers.

2. Use of aluminum trihydroxide as an abrasive agent in an airpolishing system should be avoided around the margins of cast restorations.

\section{References}

1. Barnes CM, Hayes EF, Leinfelder KF: Effects of an airabrasive polishing system on restored surfaces. Gen Dent 1987; 35:186-189

2. Gutmann, ME: Air polishing: a comprehensive review of the literature. J Dent Hyg 1998; 72:47-56

3. Cooley RL, Lubow RM, Patrissi GA: The effect of an airpowder abrasive instrument on composite resin. J Am Dent Assoc 1986; 112:362-364

4. Lubow RM, Cooley RL: Effect of an air-powder abrasive instrument on restorative materials. J Prosthet Dent 1986; 55:462-465

5. Reel DC, Abrams H, Gardner Sl, et al:: Effect of a hydraulic jet prophylaxis system on composites. J Prosthet Dent 1989; 61: 441-445

6. Eliades GC, Tzoutzas JG, Vougiouklakis GJ: Surface alterations on dental restorative materials subjected to an air- powder abrasive instrument. J Prosthet Dent 1991; 65:27-33
7. Gutmann MS, Marker VA, Gutmann JL: Restoration surface roughness after air-powder polishing. Am J Dent 1993; 6:99-102

8. Patterson CJ, McLundie AC: A comparison of the effects of two different prophylaxis regimes in vitro on some restorative dental materials. A preliminary SEM study. Br Dent J $1984 ; 157: 166-170$

9. de Boever JA, Vande Velde F: Aerosol-jet device for plaque removal: a clinical and scanning electron microscopy study. Dtsch Zahnarztl Z 1985; 40:725-729

10. Cooley RL, Brown FH, Stoffers KW: Effect of air-powder abrasive spray on glass ionomers. Am J Dent 1988; 1:209-213

11. Felton DA, Bayne SC, Kanoy BE, et al.: Effect of air abrasives on marginal configurations of porcelain-fused-tometal alloys: an SEM analysis. J Prosthet Dent 1991; 65:38-43

12. Vermilyea SG, Prasanna MK, Agar JR: Effect of ultrasonic cleaning and air polishing on porcelain labial margin restorations. J Prosthet Dent 1994; 71:447-452

13. Lehne RK, Winston AE: Abrasivity of sodium bicarbonate. Clin Prev Dent 1983; 5:17-18

14. Atkinson DR, Cobb CM, Killoy WJ: The effect of an airpowder abrasive system on in vitro root surfaces. J Periodontol 1984; 55:13-18

15. Mishkin DJ, Engler WO, Javed T, et al.: A clinical comparison of the effect on the gingiva of the Prophy-Jet and the rubber cup and paste techniques. J Periodontol 1986; 57:151-154

16. Berkstein S, Reiff RL, McKinney JF, et al.: Supragingival root surface removal during maintenance procedures utilizing an air-powder abrasive system or hand scaling: an in vitro study. J Periodontol 1987; 58:327-330

17. Christensen R: Oral prophylaxis: Prophy-Jet. Clin Res Assoc Newsl 1981; 5:1

18. de Wet FA, Holtzhausen T, Nel JJ: Evaluation of a new prophylaxis device. J Dent Assoc S Afr 1984; 39:207-208

19. Boyde A: Airpolishing effects on enamel, dentine, cement, and bone. Br Dent J 1984; 156:287-291

20. Weaks LM, Lescher NB, Barnes CM, et al.: Clinical evaluation of the Prophy-Jet as an instrument for routine removal of tooth stain and plaque. J Periodontol 1984; 55:486-488

21. Despain B, Nobis RK: Comparison of rubber cup polishing and air polishing on stain, plaque, calculus and gingiva (Abstract). Dent Hyg 1988; 62:55

22. Neme AL, Frazier KB, Roeder LB, et al.: Effect of prophylactic polishing protocols on the surface roughness of esthetic restorative materials. Oper Dent 2002; 27:50-58

23. Barnes, CM, Covey, DA, Walker MP, et al.: Essential selective polishing: the maintenance of aesthetic restorations. J Prac Hyg 2003; 12:18-24 